\title{
HERRAMIENTAS PARA LA ELECCIÓN DE DOCUMENTOS AUTÉNTICOS COMO PUNTO DE PARTIDA PARA LA CREACIÓN DE SECUENCIAS DIDÁCTICAS EN ELE
}

Ileana Arias Corrales

\section{(C) $(\oplus \Theta \Theta$}

Esta obra está bajo una licencia Creative Commons

Reconocimiento-No Comercial-Sin Obra Derivada 



\title{
HERRAMIENTAS PARA LA ELECCIÓN DE DOCUMENTOS AUTÉNTICOS COMO PUNTO DE PARTIDA PARA LA CREACIÓN DE SECUENCIAS DIDÁCTICAS EN ELE
}

\author{
TOOLS FOR CHOOSING AUTHENTIC DOCUMENTS AS \\ A STARTING POINT FOR THE CREATION OF DIDACTIC \\ SEQUENCES IN ELE
}

\section{Ileana Arias Corrales}

\begin{abstract}
RESUMEN
En este artículo se presenta, en primer lugar, la definición y tipología de documentos auténticos en el ámbito de la enseñanza de lenguas extrajeras. Seguidamente, se analizan algunas propuestas en cuanto a la pertinencia de su uso y luego se propone una serie de criterios para la elección de documentos auténticos orales o escritos como punto de partida para la planificación de una secuencia didáctica. Dicha propuesta tiene como objetivo primordial brindar herramientas y sobre todo criterios de reflexión que orienten las decisiones de la persona ya que en lo que respecta a la planificación didáctica, son las decisiones acertadas las que garantizan un curso de lengua extranjera coherente, motivante y a la vez eficaz. Finalmente, la autora presenta algunas pistas de explotación de dichos documentos.

Palabras clave: Español lengua extranjera, documentos auténticos, documentos reales, ELE, didáctica ELE.
\end{abstract}

\begin{abstract}
In this article, the author endeavors to define and describe different types of authentic materials used to teach foreign languages. Then, a few proposals regarding the use of these materials are analyzed. A set of criteria to select authentic spoken or written materials for lesson planning is also proposed with the aim of informing teachers' decision-making and contributing to their reflection on their teaching practices. Making appropriate pedagogical decisions proves to be key when trying to put together a foreign language course that is coherent, motivating and effective. Finally, recommendations for the use of authentic materials are provided.

Key words: Spanish as a foreign language, authentic documents, real documents, ELE, didactic ELE.
\end{abstract}

\footnotetext{
Mag. Ileana Arias Corrales. Universidad de Costa Rica. Docente de la Escuela de Lenguas Mordernas. Costa Rica.

Correo electrónico: ileariascorrales@hotmail.com
}

Recepción: 15- 05- 2015

Aceptación: 30- 08- 2015 


\section{Introducción}

Los documentos auténticos (DA) -o reales- son introducidos en el ámbito de la enseñanza de lenguas extranjeras a finales de los años 70, en el marco de las metodologías comunicativas. Para estas últimas el objetivo primordial es entrenar a los aprendices para que interactúen de manera eficaz en situaciones de comunicación cotidianas.

Años más tarde, tras la publicación del Marco Común Europeo de Referencia para las Lenguas europeas (MCERL) en el 2001, el uso de documentos auténticos retoma fuerza y se impone como una manera eficaz de desarrollar en el aprendiz de lengua extranjera no sólo las habilidades lingüísticas, sino también comunicativas y pragmáticas. Bajo esta perspectiva, dentro de la metodología accional el estudiante se convierte en un actor social dentro y fuera del aula de ELE, lo cual privilegia todo tipo de comunicación e intercambio real. Según la "Base de documentos a utilizar en la Didáctica de Lenguas, Soportes y Recursos Pedagógicos" (Citado por Maestro, 2003, p. 1), el objetivo de la enseñanza de lenguas extranjeras en la actualidad permite el acceso directo al conjunto de recursos auténticos escritos, orales e iconográficos a partir de los cuales los docentes planificarán la mayoría de las actividades de clase.

En cuanto a las corrientes metodológicas eclécticas actuales, cada vez más presentes en la clase de lengua extranjera, el DA constituye el punto de partida para la planificación didáctica. Como eclectismo comprenderemos la "mezcla" de rasgos metodológicos en el momento de crear, planificar y animar un curso de lengua, adaptados al contexto de enseñanza-aprendizaje. Esta "mezcla" no es intuitiva ya que parte de la idea de que el docente es el conceptor de sus dispositivos pedagógicos, es decir, de que es el autor del material didáctico que usa en clase, ya sea para sustituir el libro de texto, o para complementarlo. La premisa del eclectismo parte de la toma de decisiones acertadas del docente, lo cual conlleva una reivindicación de su autonomía. El docente que trabaja a partir del eclectismo concebido como un conjunto de decisiones y de prácticas coherentes, debe poseer un perfil de alto nivel (formación continua, experiencia, conocimiento de la actualidad, etc.) que lo dote de conocimiento y reflexión para la toma de decisiones acertadas.

Así las cosas, el hecho de confrontar al aprendiz con matrices que le son familiares o nuevas y en las cuales puede encontrar al mismo tiempo información actual y atrayente para estudiar tanto la lengua meta como la cultura meta, apuntan cada vez a trabajar con DA como punto de partida en la planificación de las clases de lengua extranjera.

Como docentes, muchas veces vemos una publicidad, escuchamos una canción o leemos un artículo que nos llama la atención y decidimos incluirlo en nuestra próxima clase, guiados muchas veces por nuestro instinto, por nuestros intereses o por la temática presente en el documento en cuestión. Sin embargo, la elección no siempre se hace a partir de criterios objetivos y válidos desde el punto de vista didáctica, de ahí que algunas veces luego de realizar una actividad de clase a partir de un DA el resultado no sea el esperado.

Este riesgo puede desvanecerse gracias a una acertada selección de DA en cuestión, a partir de criterios precisos y objetivos. En este artículo se presentan una serie de criterios y herramientas con el fin de guiar la reflexión del docente en la elección de un material auténtico que será utilizado como punto de partida para una secuencia o unidad didáctica.

\section{2. ¿Qué es un documento auténtico?}

Como documento auténtico (DA), comprenderemos aquellos que no han sido creados con objetivos pedagógicos. En el caso del español, este tipo de documentos es concebido por 
y para un contexto hispanohablante. Además de estas dos características, se deben tomar en cuenta las condiciones en que ha sido producido y los objetivos por los cuales se ha publicado o difundido en una determinada sociedad o país.

Al respecto, Besse (1987) señala que además de lo mencionado anteriormente, el DA debe ser una muestra extraída de la interacción entre hablantes nativos de una lengua. Para esto, hay que reconocer la importancia del contexto comunicativo en el que está inmerso el DA, independientemente de la matriz. Podemos afirmar entonces que la esencia de este tipo de documentos reside en que no haya intencionalidad pedagógica alguna y que, al mismo tiempo, sea una muestra social de la lengua en cuestión. Galisson (1980) prefiere el término "materiales sociales", ya que estos términos describen el contenido y la función de este tipo de material.

Maestro (2003) también rescata la importancia de reconocer lo social por encima de lo lingüístico. Para esta autora, los documentos auténticos "son textos de autores, imágenes fijas o animadas de España o de la América de habla hispana y grabaciones de voces de hispanohablantes cuya fuente de recursos será en la mayoría de los casos la de los medios de comunicación" (2003, p. 426). Para ella, los DA poseen al menos cinco características, a saber:

1. No creado para el aprendizaje del español.

2. El documento da una imagen de un uso lingüístico real.

3. Ligados a la actualidad.

4. Muestra de una realidad propia de alguno de los lugares donde se habla español.

5. No ajeno a los intereses del alumno.

Estos cinco puntos resumen lo dicho hasta ahora y señalan la importancia de que su uso se justifica también en que correspondan a los intereses de los estudiantes.

En cuanto a la noción de autenticidad, para Guillén, Alario, y Castro (1994), al hablar de "documento auténtico", se pretende excluir a los documentos fabricados (llamados también didácticos) con objetivos pedagógicos definidos. Muchas veces los docentes prefieren trabajar documentos fabricados o didácticos por temor a que los DA sean muy complejos o inadecuados para el público con el que laboran. Por estas razones, los docentes alteran los DA, con el objetivo de hacerlos más accesibles al nivel de los estudiantes o a sus objetivos.

Aunque el concepto de autenticidad sea relativo y aparezca en distintos grados en el contexto de la didáctica de lenguas extranjeras, se puede afirmar que en el momento en que se realizan alteraciones en el documento original estamos hablando de documentos semi-auténticos, no auténticos, ya que se cambió su naturaleza. Es importante mencionar que no todas las posiciones teóricas se oponen a alterar los DA. Por ejemplo, Lemeunier-Queré (2003) recomienda este tipo de alteraciones (cortar un texto, reescribirlo, agregar explicaciones de léxico, simplificarlo, corregir los errores de lengua, etc.) y las justifica siempre y cuando respondan a necesidades particulares del contexto didáctico. En el caso de DA como películas y novelas se puede justificar desde un punto de vista metodológico y práctico el hecho de trabajar ya sea capítulos, extractos o escenas, dada la naturaleza de estas matrices y la complicación que pueden representar tanto desde el punto de vista tanto de su extensión como desde su complejidad discursiva.

En cambio, Peytard (citado por Guillén, Alario, y Castro, 1994, p. 94) sostiene que un documento es auténtico en tanto mantenga su integridad e integralidad, por lo tanto las modificaciones no son recomendables. Según este autor, al trabajar con un documento real se busca que éste conserve las características y marcas propias de sus condiciones de producción y de recepción al igual que de la cultura en la que fue creado. 


\subsection{Tipología}

La gama de documentos auténticos es casi infinita así como sus aplicaciones para la clase de lengua extranjera. Algunas matrices comunes son las siguientes: artículos de prensa, caricaturas, publicidades, recetas, poemas, novelas, cuentos, facturas, clasificados, blogs, foros, sitios de Internet para realizar compras, correos electrónicos, tarjetas postales, mapas, panfletos, pinturas, fotografías, canciones, conferencias, noticias, programas de televisión, anuncios y películas, seguidos de un larguísimo etcétera.

Al leer esta lista, podemos hacer la primera gran clasificación en documentos propios del canal oral (programas televisivos, entrevistas, canciones, etc.) y los que son propios al canal escrito (afiches, recetas, panfletos, etc.). Asimismo, hay documentos desprovistos de texto, como lo son las fotografías o las pinturas. A este último tipo de textos lo llamaremos documentos icónicos. Por otra parte, existen documentos auténticos sonoros tales como un silbido, un ruido, etc., los cuales son difíciles de clasificar.

Nos referiremos a partir de ahora a dos tipos de DA, a saber los escritos y los orales. Dichos documentos se pueden subdividir en dos grandes grupos, los pragmáticos y los no pragmáticos. El primer grupo se refiere a aquellos que tienen una función comunicativa concreta y definida, en los cuales hay un intercambio de información, como en el caso de las conversaciones telefónicas, los correos electrónicos, y otros; por su parte, el segundo grupo hace referencia a otro tipo de expresión verbal, dentro de los cuales encontramos los del plano estético y artístico (poemas, novelas, cuentos, etc.) u oral (narraciones de cuentos, declamaciones de poemas, etc.).

Otro criterio que nos puede guiar al clasificar los DA es la intención que motiva la emisión de un mensaje en particular. Cuando se escribe o se habla, siempre hay una intención comunicativa: podemos informar, explicar, narrar, describir, persuadir o argumentar. Estos criterios pueden ser de gran utilidad sobre todo cuando se trabaja con artículos escritos.

\section{3. ¿Por qué usar documentos auténticos en la clase de ELE?}

Hay muchas razones que legitiman el uso de DA en la clase de lengua extranjera. A continuación se presentan cinco de ellas.

\subsection{Enseñar a comunicar}

Uno de los mayores retos en la enseñanza de lenguas extranjeras es el de proponer actividades en las que la lengua meta esté al servicio de la comunicación. Sabemos que enseñar un "saber" (contenido) no es lo mismo que enseñar un "saber hacer" (aplicar el saber de manera eficaz) particular. Asimismo, sabemos que en la actualidad hay un sinfín de manuales de enseñanza universalistas que preparan a los aprendices para que se desenvuelvan en situaciones comunicativas poco adaptadas a la realidad y, por ende, los libros de texto son insuficientes.

Para hacer frente al desafío que consiste en entrenar a los aprendices para que sean eficaces al interactuar en diversas situaciones comunicativas en el mundo real, el DA es un recurso esencial. La razón de ello es que muchas matrices permiten que el estudiante conozca las reglas sociales del uso de la lengua meta, los contextos de comunicación, el lenguaje no verbal y evidentemente todo lo relativo al dominio lingüístico. 


\subsection{Descubrir la actualidad en LE}

Gracias a los DA, los estudiantes conocerán y se informarán acerca de temas de actualidad, muchas veces en tiempo real, gracias a la tecnología con la que contamos actualmente. La actualidad puede ser estudiada a través, por ejemplo, de textos orales y escritos de naturaleza periodística, en los cuales se tratan temas del mundo hispano y de otras latitudes del globo.

El estudio de la actualidad da pie al desarrollo del sentido crítico de los estudiantes y abre ricos espacios de intercambio y reflexión en el aula. Para ello, además de estudiar material proveniente de los medios de comunicación, no olvidemos que los textos literarios así como las películas, canciones, blogs, fórums y escritos de opinión -por citar solamente algunas matrices- proveen información sobre la actualidad latinoamericana y mundial usando como código el español.

\subsection{Descubrir la cultura meta y trabajar la interculturalidad}

Trabajar matrices variadas conduce innegablemente a una aproximación y a una reflexión sobre la cultura meta. Gracias a los DA, los estudiantes de español como lengua extranjera tienen a su disposición una serie de material que les permitirá conocer distintas realidades del mundo hispano, de sus hablantes, de sus costumbres, de comportamientos y de sus valores.

Sin duda alguna, abordar la cultura en la clase de ELE resulta mucho más objetivo por medio del estudio de DA que si se parte de documentos fabricados para la enseñanza de lenguas. En estos últimos el docente se puede topar con puntos de vista parcializados y generalizados en los cuales hay representaciones y estereotipos sobre un país, una cultura o grupo social en particular.

Enfrentar al estudiante a una nueva realidad tendrá grandes beneficios no solo para el aprendizaje de la L2, sino también para que reflexione acerca de su propia cultura. Si un DA es elegido a partir de una serie de criterios bien establecidos, dará pie para que además de trabajar temas culturales, se den ricas discusiones referentes a temas interculturales.

Mediante el estudio de DA, el estudiante puede redescubrir su propia cultura partiendo de sus experiencias previas o de experiencias interculturales. Un ejemplo concreto es el de las recetas. En muchas lenguas, al explicar una receta siempre se va a partir de los ingredientes para describir, mediante distintas estrategias gramaticales, la preparación del plato en cuestión. En este caso en particular como en otras matrices, el estudiante puede partir de lo conocido para comprender lo desconocido.

\subsection{El corpus lingüístico como muestra real de la lengua}

Gracias a los DA, los estudiantes pueden descubrir, comprender y analizar cómo funciona la lengua meta, pueden aprender acerca de los usos, de la norma y de las variantes existentes a través de una muestra. La variedad de corpus tanto orales como escritos presentes en las matrices de DA pueden ser utilizados en clase de muchas maneras.

\subsection{Motivación e intereses de los estudiantes}

La variedad de matrices existentes y su calidad estética son esenciales para despertar la motivación de los estudiantes por la lengua meta. Según Ferrer (s.f.), "El lema que debe guiarnos es el siguiente: «Lo visual, lo tangible siempre llama la atención y mantiene vivo 
el interés de los alumnos»" (p. 2354). Proponer material actual y atractivo puede resultar altamente estimulante para los estudiantes e incidir en su implicación en el aprendizaje de la lengua meta. Asimismo, si partimos de sus intereses al elegir los DA, se puede desarrollar la curiosidad por descubrir el mundo hispanohablante.

\section{Criterios para la selección de materiales auténticos}

En esta sección se presentarán tres propuestas en relación con los criterios de selección de los DA. Al final de esta sección, se propondrán dos herramientas para la descripción y análisis de este tipo de recursos.

El principal de los criterios a retener es el de funcionalidad. Todo DA que sea elegido como documento de partida para la planificación didáctica, deberá tener esta característica. Luego, aparecen criterios presentados por distintos autores que son oportunos de rescatar. A continuación, se presentan los más importantes.

En primer lugar, para Lemeunier-Queré (2003) los criterios que deben guiar la elección del DA parten tanto de las necesidades de los estudiantes como del contexto de la clase en particular. Esto se puede observar en la siguiente lista:

- ¿La introducción del documento en el contexto de clase es legítima?

- ¿El perfil de mis estudiantes me permite utilizar este tipo de recurso?

- ¿El documento auténtico empata con el objetivo de mi lección?

- ¿La naturaleza del documento (oral, escrito, gráfico, pictórico, estrictamente auténtico o manipulado)?

- ¿La proporción del documento auténtico que uso en clase se justifica?

- ¿Qué tipo de explotación permiten los documentos auténticos?

De esta propuesta cabe rescatar la importancia que la autora le da al criterio relativo a la legitimidad del uso de los DA en clase. Utilizar este tipo de recurso no puede ser el resultado de una selección a la ligera; por el contrario, debe haber una reflexión previa orientada hacia la adecuación con los objetivos de aprendizaje y con las características del público.

En segundo lugar, Tabernero (2011) propone una serie de criterios planteados desde el punto de vista de los aprendices, lo que nos recuerda que en las metodologías actuales los docentes toman decisiones en función del estudiante de sus necesidades e intereses, pues el aprendizaje se centra en él y en su autonomía. El autor da importancia al contexto; para elegir acertadamente un DA, enumera los siguientes criterios:

- Adaptación a la edad

- Adaptación al nivel lingüístico

- Adaptación a las necesidades lingüísticas

- Adaptación a los intereses

- Adaptación a los programas

- Adaptación a las particularidades culturales

- Fiabilidad del documento

Aunque esta propuesta se asemeja a la de Lemeunier-Queré (2003), se ha de rescatar el último criterio, el de fiabilidad del documento. En una era en la que Internet se convierte en la herramienta de trabajo por excelencia, debemos preguntarnos si los documentos que buscamos son fiables desde el punto de vista de la información que transmiten. 
En tercer lugar, presentamos la propuesta de Valérie Lemeunier (2003), por mucho la más completa y compleja. A continuación, se exponen los ocho criterios propuestos por esta autora: ${ }^{1}$

1. ¿Está adaptado al público meta?

¿En relación a su edad?/ ¿En relación a su nivel de lengua?/ ¿En relación a sus centros de interés? ¿En relación a su herencia sociocultural? (historia individual y colectiva)/ ¿En relación a sus hábitos?/ ¿En relación a sus objetivos?/ ¿En relación a sus necesidades lingüísticas?

2. ¿Hay elementos no verbales que faciliten la comprensión?

¿La fuente es conocida?/ ¿La fecha es importante?/ ¿El autor / locutor es conocido?/ ¿El título y el paratexto dicen algo?

3. ¿Los parámetros de la situación de la comunicación aparecen claramente?

La naturaleza del documento: ¿De qué se trata?

Hablantes: ¿Quién habla o escribe? ¿A quién?

Lugar de la interacción: ¿Cuándo tiene lugar la escena?

Manera en que se desarrolla la interacción: ¿Cómo se desarrollan los intercambios?

Causa de la interacción: ¿Por qué razón tiene lugar el intercambio?

Desafío presentado en la interacción: ¿Cuál es el objetivo del intercambio?

4. ¿El documento se presta para ser explotado pedagógicamente?

¿Hay suficientes elementos para emitir hipótesis antes de leer o escuchar el documento?

¿La verificación de las hipótesis puede hacerse gracias a una escucha o lectura rápida o parcial? ¿Hay contenido suficiente para llevar a cabo una comprensión detallada en un marco auténtico?

5. ¿El contenido comunicativo es pertinente en relación a la progresión establecida? ¿Está adaptado al o los objetivo(s) comunicativo(s) planteado(s)?/ ¿Permite reactivar los saberhacer estudiados con anterioridad?/ ¿Permite sensibilizar a objetivos futuros?/ ¿Presenta un buen corpus para la búsqueda de información y la conceptualización?

6. ¿El contenido lingüístico es pertinente en relación con la progresión?

¿Está en adecuación con las estructuras por adquirir?/ ¿Permite reactivar estructuras estudiadas anteriormente?/ ¿Permite sensibilizar a nuevas estructuras?/ ¿Presenta un buen corpus para la búsqueda de información y la conceptualización?

7. ¿El contenido sociocultural es pertinente?

¿Es un buen reflejo de la sociedad francesa (o francófona)?²/ ¿Permite una pedagogía intercultural?/ ¿Está en adecuación con los conocimientos por adquirir?/ ¿Permite reactivar conocimientos anteriores?/ ¿Permite sensibilizar a los conocimientos por estudiar en el futuro?/ ¿Es interdisciplinario?/ ¿Permite reutilizar los conocimientos adquiridos fuera de la clase y viceversa?

8. ¿El documento es un buen documento de partida?

¿Para una sistematización oral?/ ¿Para una sistematización escrita?/ ¿Para una producción oral?/ ¿Para una producción escrita?

Según la autora, a mayor número de respuestas afirmativas, más pertinente será el DA. Además, señala la importancia de que exista una coherencia entre el marco metodológico con el que trabaja el docente y el documento elegido. 
A pesar de que no exista un material auténtico ideal y de que evidentemente nos topemos con dificultades en algunas matrices debido a la extensión, dificultad léxica, connotaciones culturales confusas y otras razones, estos criterios son muy acertados en el momento de escoger un DA. Asimismo, cabe aclarar que cada matriz tiene sus propios elementos de pertinencia y de explotación, por lo tanto una canción no se abordará de la misma manera que un texto periodístico.

Con sus criterios de pertinencia, Lemeunier (2003) logra incluir los planos lingüístico, pragmático, sociolingüístico, comunicativo; además, toma en cuenta el contexto, los intereses y las características del aprendiz. Esta propuesta se basa en criterios de pertinencia que van más allá de una simple verificación de lo que se puede adaptar o no a un contexto en particular. Los criterios presentados nos hacen reflexionar sobre la complejidad de la elección de un DA y sobre el amplio panorama de los elementos que pueden influir en los procesos de enseñanzaaprendizaje de una lengua extranjera.

A partir de los criterios mencionados anteriormente, a continuación se propone una herramienta que retoma todos estos elementos y los sistematiza en dos tablas. La primera de ellas tiene como objetivo que el profesor tenga acceso a una ficha resumen sobre las principales características del DA en cuestión. Dicha ficha es general y puede completarse para cualquier tipo de material, siendo esta un punto de partida en el análisis prepedagógico de un DA.

Tabla 1

\section{Descripción del Documento auténtico}

Fuente:

Autor/ locutor: Nivel (es) $\square$ A1 $\square$ A2 $\square \mathrm{B} 1 \square \mathrm{B} 2 \square \mathrm{C} 1 \square \mathrm{C} 2$

Tipo de matriz:

Tipo de soporte:

Documento: $\square$ Oral $\square$ Escrito $\square$ Iconográfico $\square$ Otro

Documento oral/ audiovisuales:

- Duración:

- Elementos no verbales:

Documentos escritos:

- Extensión:

- Elementos paratextuales presentes:

Objetivos de aprendizaje por cumplir por medio de la didactización del documento:

Contenidos presentes en el corpus/paratexto:

$\square$ Léxicos:

$\square$ Gramaticales:

$\square$ Fonéticos:

$\square$ Sociolingüísticos:

$\square$ Pragmáticos:

$\square$ Culturales:

Otros: 
En la primera parte de esta ficha se puede introducir la información general del documento para que de esta manera el docente pueda encontrar fácilmente la referencia en caso de que sea necesario o bien para mutualizar esta información con sus colegas. El tipo de matriz se refiere al tipo de documento en sí (publicidad, receta, carta de renuncia, canciones, etc.) y el tipo de soporte se refiere al documento en físico, es decir, si se trata de un texto impreso, digital, en formato MP3, etc. También, se propone una sección en la que el docente puede anotar si se trata de un documento oral o escrito y si están en concordancia con los objetivos o tareas que debe cumplir. Luego, se presenta una sección en la que se puede anotar el tipo de contenidos presentes en el DA, teniendo en cuenta que un documento idóneo debería cubrir todos estos contenidos. Finalmente, se plantea una sección denominada "otros", útil para anotar cualquier otra información que sea pertinente en relación a la descripción del DA.

Seguidamente, proponemos la segunda tabla para elegir acertadamente un DA. Esta resume la mayoría de los criterios expuestos principalmente por Lemeunier (2003), y se agregan otros que no habían sido contemplados explícitamente por la autora.

Tabla 2

CRITERIOS PARA LA ELECCIÓN DE UN DA

\begin{tabular}{|l|c|c|}
\hline 1. Estética del documento & Sí & No \\
\hline 1.1 ¿El documento es legible/audible? & & \\
\hline 1.2 ¿El documento es atractivo/llama la atención? & & \\
\hline
\end{tabular}

\begin{tabular}{|l|c|c|}
\hline 2. El contexto de enseñanza & Sí & No \\
\hline 2.1 ¿El documento está adaptado a la programa/progresión de la institución? & & \\
\hline $\begin{array}{l}2.2 \text { ¿El documento está adaptado a las características de los estudiantes? (¿edad, intereses, } \\
\text { nivel lingüístico?) }\end{array}$ & & \\
\hline 2.3 ¿El documento parte de experiencias previas del estudiante? & & \\
\hline
\end{tabular}

\begin{tabular}{|l|l|l|}
\hline 3. La explotación pedagógica del documento & Sí & No \\
\hline 3.1 ¿El documento puede ser explotado pedagógicamente? & & \\
\hline 3.2 ¿El documento se adecua a los objetivos/tareas del curso? & & \\
\hline 3.3 ¿El documento es un buen documento de partida? & & \\
\hline 3.4 ¿El documento permite formular hipótesis sobre su contenido? & & \\
\hline 3.5 ¿El documento presenta elementos no verbales que faciliten la comprensión? & & \\
\hline 3.6 ¿El documento permite desarrollar habilidades comunicativas? & & \\
\hline 3.7 ¿El documento me permite desarrollar actividades diversas? & & \\
\hline 3.8 ¿El documento tiene un corpus lingüístico que puede ser explotado posteriormente? & & \\
\hline 3.9 ¿El contenido lingüístico (gramatical, léxico, fonético) es pertinente? & & \\
\hline $3.10 ~ ¿ E l$ contenido sociolingüístico es pertinente? & & \\
\hline 3.11 ¿El contenido cultural es pertinente y objetivo? & & \\
\hline $3.12 ~ ¿ E l$ documento permite sensibilizar al estudiante a variantes de la lengua meta? & & \\
\hline 3.13 ¿El documento permite abordar temas estudiados anteriormente? & & \\
\hline 3.14 ¿El documento permite abordar temas por estudiar? & & \\
\hline
\end{tabular}


Esta tabla no pretende ser una herramienta finalizada, es más bien un recurso en construcción. Con experiencia en la materia, el docente podrá hacer sus aportes y modificaciones a esta base.

\section{Pistas de didactización}

Luego de elegir el DA, el docente pasará a la etapa de planificación didáctica. Aunque el objetivo de este artículo no es presentar una metodología en particular para didactizar DA, se abordará el tema por medio de algunas pistas que permitan al docente crear su propia manera de abordarlos.

En primer lugar, debemos tomar en cuenta tanto el grado de dificultad como la extensión o duración del documento. Como se señaló anteriormente, si usamos un DA como documento de partida, desde el punto de vista metodológico privilegiaremos los enfoques orientados a la interacción real y al cumplimiento de tareas. Por esta razón, se propone que haya un mínimo de tres etapas a seguir en el momento de planificar las actividades de clase entorno al DA, independientemente de que el documento en cuestión sea utilizado para planificar actividades de comprensión o de sistematización de un contenido linguiístico en particular:

Tabla 3

\begin{tabular}{|c|c|}
\hline Documento oral-audiovisual & Documentos escritos \\
\hline Actividad de pre escucha & Actividad de pre lectura \\
Actividad de escucha & Actividad de lectura \\
Actividad de post escucha & Actividad de post lectura \\
\hline
\end{tabular}

Tomando en cuenta que los documentos orales son muy distintos de los escritos tanto en sus matrices como en las técnicas y estrategias utilizadas para su comprensión, partimos de la idea de que toda presentación de un DA debe ser precedida de una actividad introductoria que permita emitir hipótesis sobre el contenido del material. Posteriormente, se presentan actividades para comprender el documento por medio, ya sea de la escucha o de la lectura. Cabe aclarar que el docente y los estudiantes negociarán cuántas lecturas/escuchas son necesarias, todo depende de la planificación de las actividades. Finalmente, se debe proponer una actividad ya sea de reempleo o de extensión, en la que no esté involucrada la comprensión, sino otras habilidades comunicativas.

Para Lemeunier (2003), la explotación del documento parte de la comprensión para llegar a la producción, respetando la naturaleza del DA y su finalidad comunicativa. Por ejemplo, si la matriz que se usó para iniciar la unidad didáctica fue la grabación de una reservación vía telefónica, se espera que los aprendices produzcan una conversación similar a ésta una vez que se hayan apropiado de las nuevas estructuras y contenidos tanto lingüísticos como sociolingüísticos y pragmáticos. Si el estudiante es eficaz en la etapa de producción, los objetivos de aprendizaje han sido alcanzados. Bajo esta perspectiva, las etapas son las siguientes:

1. Sensibilización

2. Comprensión:
a. Anticipación (acceso al sentido, emisión de hipótesis)
b. Comprensión global (verificación de hipótesis, preguntas orientadas hacia los parámetros comunicativos)
c. Comprensión detallada 
3. Tratamiento lingüístico:

a. Búsqueda (el estudiante es un detective, busca en el corpus del DA la información relevante para analizar la lengua y para proponer reglas de funcionamiento)

b. Conceptualización (el estudiante negocia para proponer una regla definitiva)

c. Sistematización (ejercicios variados para poner en práctica los contenidos nuevos)

4. Producción o reempleo

Está claro que el éxito de la explotación de un DA va a depender tanto de la elección del documento como de la guía que el docente proporcione. Este enfoque ha sido criticado sobre todo en la etapa referente al tratamiento lingüístico, pues muchas veces el estudiante no es capaz de comprender un contenido gramatical solamente observando. Sin embargo, este enfoque es muy eficaz si el estudiante se acostumbra a su funcionamiento. Igualmente, hay que reconocer que esta propuesta es acorde con lo propuesto en las metodologías actuales en las cuales el estudiante es autónomo y consciente de su aprendizaje.

\section{Recomendaciones finales}

Como recomendaciones finales, al trabajar con DA es importante tener en cuenta los siguientes puntos:

- $\quad$ El docente debe ser coherente y pertinente en las propuestas pedagógicas que presente.

- Las matrices de DA deben ser variadas y pertenecer tanto al canal oral como al escrito.

- Al trabajar el DA es imperativo desarrollar tanto las habilidades lingüísticas como las comunicativas, en todos los niveles de enseñanza de ELE, desde nivel A1 a nivel C2.

- Es conveniente privilegiar un enfoque inductivo en el que el estudiante sea activo y descubra por sí mismo, las reglas de funcionamiento de la lengua extranjera.

- Se sugiere tomar en cuenta que cada matriz exige distintos procesos de comprensión, de estrategias y de técnicas que deben ser facilitadas por el docente.

- La variedad de actividades que proponga el docente alrededor del DA, harán su propuesta aún más eficaz y significativa para los aprendices.

- Aunque los DA sean elegidos con objetividad, muchas veces no son bien acogidos por los estudiantes. El docente debe saber que está expuesto a riesgos y que no siempre podrá satisfacer las expectativas de los aprendices.

- Se recomienda tener siempre presente que el trabajo con DA conlleva comprender un código nuevo (L2) e interpretar connotaciones e implícitos culturales, lo que no es sencillo.

- Finalmente, es importante mencionar que trabajar un DA siguiendo los lineamientos presentados anteriormente no es una tarea nada simple; sin embargo, los resultados de su aplicación son más que satisfactorios.

\section{Conclusiones}

En guisa de conclusión, es necesario recordar que el uso de un DA como documento de partida es una labor compleja si se desea hacer de manera acertada. Gracias a la variedad de matrices existentes, el material auténtico resulta de gran utilidad para el desarrollo de todo tipo de habilidades en lengua extranjera; además, beneficia el desarrollo del saber ser y del saber aprender. 
Actualmente, en disciplinas como el francés lengua extranjera (FLE), cada vez más instituciones de enseñanza han optado por no utilizar más libros de texto y proponer a los docentes que seleccionen DA y los didacticen en función de sus programas de enseñanza, siendo el DA el protagonista. Sin duda, esta práctica conlleva grandes desafíos profesionales, ya que de cierta manera hace que el docente asuma una función que va más allá de transmitir contenidos lingüísticos; bajo esta perspectiva el docente es un conceptor de material didáctico.

Trabajar con DA nos permite mostrar al estudiante la actualidad y temas culturales de gran interés; de igual manera estos recursos hacen reflexionar al estudiante sobre el funcionamiento de la sociedades, sobre la manera en que crean y transmiten información.

Finalmente, es necesario aclarar que no existe un enfoque ideal en lo que respecta los DA. El profesor de lengua extranjera tendrá el poder de decisión al trabajar con este recurso, eso sí, respetando siempre su autenticidad, lo que hace único este recurso.

\section{Notas}

1. Traducción propia.

2. En este caso, adaptaremos este criterio a "sociedades hispanohablantes".

\section{Bibliografía}

Besse, H. (1987). Documents authentiques et enseignement/apprentissage de la grammaire d'une LE. Paris: Didier.

Ferrer, C. (s.f.). Reflexiones sobre el uso de documentos auténticos en la clase de ELE. http:// www.letras.ufmg.br/espanhol/Anais/anais_paginas_\%2020102501/Reflexiones\%20 sobre.pdf [Consulta 27 de febrero de 2015].

Galisson, R. (1980). D’hier à aujourd'hui la didactique générale des langues étrangères. Paris: Clé International.

Guillén, C., Alario, I. y Castro, P. (1994). Los “documentos auténticos” en la renovación metodológica del área de lengua extranjera. Comunicación, Lenguaje y Educación. 24, 89-100.

Lemeunier-Queré, M. (2003). Créer du matériel didactique: un enjeu et un contrat. http://www. edufle.net/Creer-du-materiel-didactique-un.html [Consulta 07 de marzo de 2015].

Lemeunier, V. (2003). Élaborer une unité didactique à partir d'un document authentique. http://www.francparler-oif.org/pour-lenseignant/les-articles/2645-elaborer-une-unitedidactique-a-partir-dun-document-authentique.htmluthentique [Consulta 12 de febrero de 2015].

Maestro, A. (2003). Los medios de comunicación: el gran canal de "documentos reales" en el proceso de enseñanza-aprendizaje del español lengua extranjera. XIV Congreso Internacional de ASELE. Universidad de Burgos. http://cvc.cervantes.es/ensenanza/ biblioteca_ele/asele/pdf/14/14_0426.pdf. [Consulta 12 de febrero de 2015].

Tabernero, C. (2011). Atelier: créer des supports d'enseignement à partir de documents authentiques. Actes du XIlème colloque pédagogique de l'Alliance française de São Paulo. (1-14). Alliance française de São Paulo. 